\title{
Aging and epigenetic drift: a vicious cycle
}

\author{
Jean-Pierre Issa
}

Fels Institute for Cancer Research, Temple University School of Medicine, Philadelphia, Pennsylvania, USA.

\begin{abstract}
The term epigenetics refers to stable patterns of gene expression that are seen during differentiation or $\mathrm{X}$ chromosome inactivation and are not dependent on dynamic changes in coding DNA. These gene expression states are encoded in the epigenome - a collection of marks on DNA or on histone tails that are established during embryogenesis. Genome-wide studies in aging cells and tissues have uncovered stochastic DNA methylation drift (gradual increases or decreases at specific loci) that reflects imperfect maintenance of epigenetic marks. Drift creates epigenetic mosaicism in aging stem cells that could potentially restrict their plasticity and worsen phenotypes such as stem cell exhaustion and focal proliferative defects that can lead to cancer.
\end{abstract}

\section{Epigenetics}

The term epigenetics arose from a need to explain cellular phenotypic diversity in the face of a shared genetic make-up, as observed during differentiation in multicellular organisms (1). This is an extreme form of gene expression regulation, in which stability is key. Just as a lymphocyte does not spontaneously become an epithelial cell, an epigenetic pattern is stable through multiple rounds of cell division. While the concept arose to explain differentiation, epigenetic phenomena soon expanded to include other stable forms of gene expression regulation such as $\mathrm{X}$ chromosome inactivation in females (2) and imprinting, whereby a few hundred genes are expressed from only one of the two inherited alleles in a parent-of-origin manner (3). Our understanding of epigenetics has grown by leaps and bounds in the past decades as the mystery of stable gene expression in the absence of genetic change was solved through the discovery of DNA methylation- and chromatin-based gene regulation.

DNA methylation refers to the covalent addition of a methyl group (CH3) to a DNA base (4). In mammals, only cytosine can be normally methylated, and this most often happens in the context of the symmetrical dinucleotide CG (often referred to as $\mathrm{CpG}$ ). About half of human promoters and transcription start sites are embedded in $\mathrm{CPG}$ islands (discrete regions rich in $\mathrm{CPG}$ sites and about 0.5 to 2 kilobases in length), and about half of all $\mathrm{CpG}$ islands are gene promoter associated. The 5-methylcytosine base can be further modified through sequential carboxylation by the ten-eleven translocation (TET) family of enzymes (5). These recently discovered modifications (e.g., 5-hydroxymethylcytosine) are present at low levels in the genome and are intermediates in a reaction that eventually leads to demethylation.

A link between DNA methylation, gene expression, and epigenetics was proposed more than 30 years ago (6), and considerable evidence has since accumulated to confirm this link. DNA methylation shows unique patterns associated with physiologic epigenetic states; it is tissue specific, distinctly different on the inactive $\mathrm{X}$ chromosome compared with the active $\mathrm{X}$ chromosome, and different between expressed and silenced imprinted alleles. Genetic (7) or pharmacologic (8) disruption of DNA methylation interferes with proper epigenetic regulation, demonstrating that the link is causal; however, that link is also complex, in that the role of DNA methylation in gene expression depends on the CPG context $(9,10)$. Promoter methylation is associated with gene silencing,

Conflict of interest: Jean-Pierre Issa is a paid consultant for, and has received research support from, Astex Pharmaceuticals Inc.

Citation for this article: J Clin Invest. 2014;124(1):24-29. doi:10.1172/JCI69735. gene body methylation has variable effects on gene expression, and intergenic methylation may also affect expression through enhancer regulation. There is no doubt that promoter CpG island methylation is a true epigenetic mark - it is stable and self-perpetuated through cell division by the maintenance methylase DNA methyltransferase 1 (DNMT1) (4). DNA methylation in other contexts can be more dynamic and sometimes follows rather than causes gene expression changes, and thus may not always have the stability required to maintain a true epigenetic state.

Posttranslational modification of histone tails provides another level of gene expression control (11). Long thought to be inert structural proteins around which DNA is wrapped, histones have emerged as key players in both transient and long-term gene expression regulation. Histone modifications are established by "writers" that catalyze the transfer of acetyl groups, methyl groups, or other moieties to a limited set of amino acids that protrude from the nucleosome and serve as signaling molecules. These modifications trigger binding by various proteins (readers) that interpret the signal and either repress or activate gene expression, generally by inducing local compaction or relaxation of chromatin through movement of nucleosomes, respectively. Long-term gene silencing is associated with distinct histone modifications (12), leading to the idea of epigenetic regulation by these modifications. However, enzymes that reset posttranslational modifications (editors) are abundant in adult cells, and histone modifications can change rapidly in response to cellular environments (13). Some have argued that the dynamic nature of histone modifications make them poor candidates to explain epigenetic states (14).

Mechanisms that mediate epigenetics have generated considerable interest in the past decade, and some confusion has arisen over the definition of epigenetics. The term has sometimes been used to encompass all chromatin states, including some unrelated to gene expression (e.g., DNA repair) (15). It is also used to indicate pathway deregulation by gene expression (as opposed to mutations), regardless of the ultimate mechanism (16). These expanded uses of the word move away from an essential component of epigenetics - stability. Gene expression induction by a changed cellular state such as proliferation or a stress response may involve chromatin remodeling or even DNA demethylation, but it is fundamentally different from gene expression changes during differentiation. Epigenetic silencing for genes on the inactive $\mathrm{X}$ chromosome or for imprinted genes occurs despite adequate cellular levels of the transcription factors that regulate the target genes. Mechanistically, it is important to distinguish stable expression (epigenetic) from dynamic expression (often transcription factor based), but there is an incomplete 
A

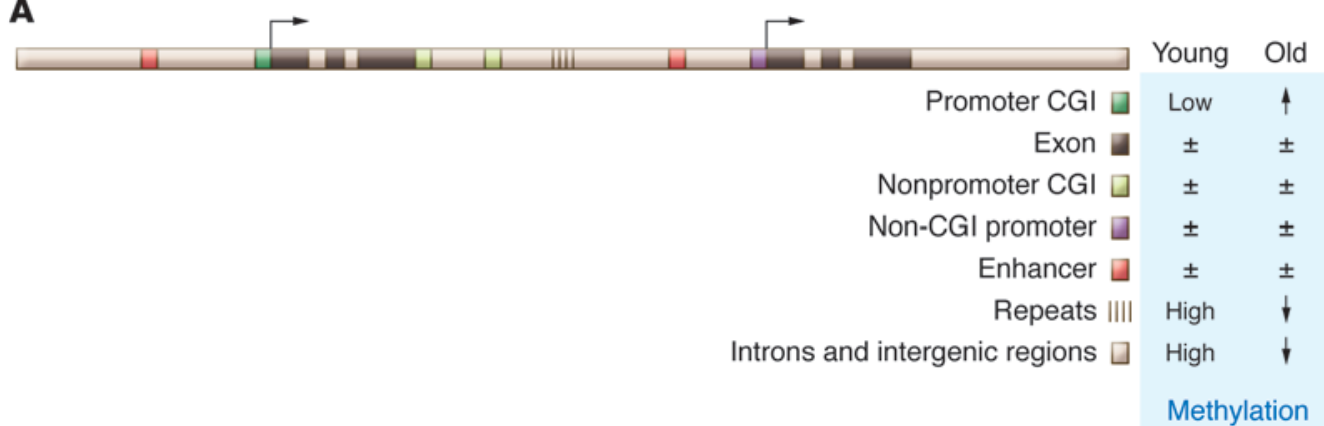

B

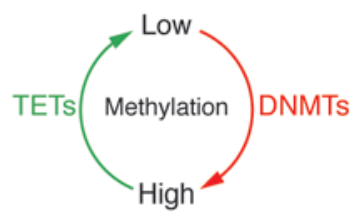

\section{Figure 1}

Dynamics of DNA methylation in genomic compartments. (A) The top line represents DNA containing two genes (arrows indicate transcription start sites; exons are shown in black); three CpG islands (CGl; green) located in a promoter, a 3' end, and an intergenic area; two enhancers (red); a series of repeats (thin black lines); a non-CGI promoter (purple); and intergenic and intronic DNA (open boxes). The normal methylation state and aging changes are summarized in the light blue box. (B) Effector enzymes that switch DNA methylation on or off. DNMTs include DNMT1, DNMT3a, and DNMT3b; TETs include TET1, TET2, and TET3.

understanding of which cellular mechanisms other than $\mathrm{CpG}$ island promoter methylation are truly unique to stable epigenetic states. In the following sections, I will use the term epigenetics to refer to stable gene expression regulation.

\section{DNA methylation changes in aging cells and tissues}

DNA methylation was first measured globally using biochemical methods, and early studies showed progressive depletion of 5-methylcytosine associated with in vitro senescence in normal fibroblasts (17). Similar changes were observed in aging mouse tissues and human cells (18), though the changes were quite small (from $\sim 4.05 \%$ to $3.95 \%$ over seven decades) (19). Another estimate of global methylation can be derived by studying normally methylated repetitive elements such as Alu or LINE1 elements. These studies have yielded inconsistent results $(20,21)$, and differences may be tissue specific. The methylation state of individual genes can be studied by taking advantage of two properties of methylated CpG sites: methylation blocks the activity of many restriction enzymes, and a methylated cytosine is relatively resistant to deamination by treatment with bisulfite. Both methods allow the quantitative interrogation of individual CPG sites (22). Early studies focused on promoter $\mathrm{CpG}$ islands, particularly those that showed a marked increase in methylation associated with cancer. Southern blot analysis showed that the estrogen receptor $\alpha(E R a)$ promoter is unmethylated in tissues of young individuals, but gains methylation at a rate of $1 \%$ every 3 years in human colon (23). Many genes were subsequently shown to behave similarly (24), including 70\%-80\% of promoter CpG islands that show hypermethylation in cancer (25). In tissue collected at autopsy, hypermethylation was observed in most tissues, with substantial tissue-to-tissue variability (26). In the intestine, microdissection and analysis of individual crypts showed considerable variation in age-related methylation, with much higher intercrypt than intracrypt heterogeneity (27). Given that each colonic crypt is derived from a single or very few stem cells, the data suggest that age-related hypermethylation is a property of stem cells.

Genome-wide technologies to study DNA methylation have facilitated studies of aging and revealed striking and reproducible changes that are dependent on genomic context (summarized in Figure 1). In the mouse, almost a quarter of the queried CPG sites showed age-related methylation changes that could be found in all tissues, with the most prominent changes observed in the most proliferative organs such as the gastrointestinal tract and spleen (28). Similar replication-dependent changes were seen in hematopoietic stem cells (29). Precise quantitation revealed that methylation changed progressively and linearly with age, and that hyper- and hypomethylation occurred at comparable rates. Thus, the changes are most consistent with drift (a gradual change away from baseline) rather than an abrupt or programmed phenomenon. Studies of human tissues also revealed numerous changes (both hyper- and hypomethylation) (30-33). It has been proposed that one can estimate a person's age by looking at DNA methylation in peripheral blood or in other tissues (34). Although tissue specific overall (34), some genes show conserved patterns in different tissues, and it might be possible to use drift in peripheral blood as a surrogate for drift in other tissues (35).

As described above, a methylation change is simply defined as a difference between two states, in this case old versus young. It does not imply primary pathology or functional consequences. To address this issue, one has to consider the particular $\mathrm{CpG}$ sites involved and other physiologic changes that accompany aging. Given that methylation is tissue specific, shifts in cellular composition or differentiation state of a tissue can be associated with alterations in methylation. Genome-wide studies have shown shifts in global methylation associated with stem cell differentiation (9), and it is possible that shifts in 5-methylcytosine content are related to physiologic changes in proliferation $(36,37)$. Thus, some of the age-related methylation drift could in fact be a secondary consequence of small differences in differentiation or proliferation states. It may be possible to deconvolute pathologic changes from shifts in cellular content using recently described algorithms based on differential gene methylation (38) or expression (39). However, more than $97 \%$ of promoter-associated CPG islands are unmethylated in normal cells and tissues from young individuals (40), regardless of differentiation of proliferation. Thus, the common age-related changes observed in this genomic compartment likely represent pathologic events rather than altered tissue composition.

\section{Histone modification changes}

Posttranslational histone modifications are both dynamic and somewhat difficult to measure quantitatively. This poses a special 


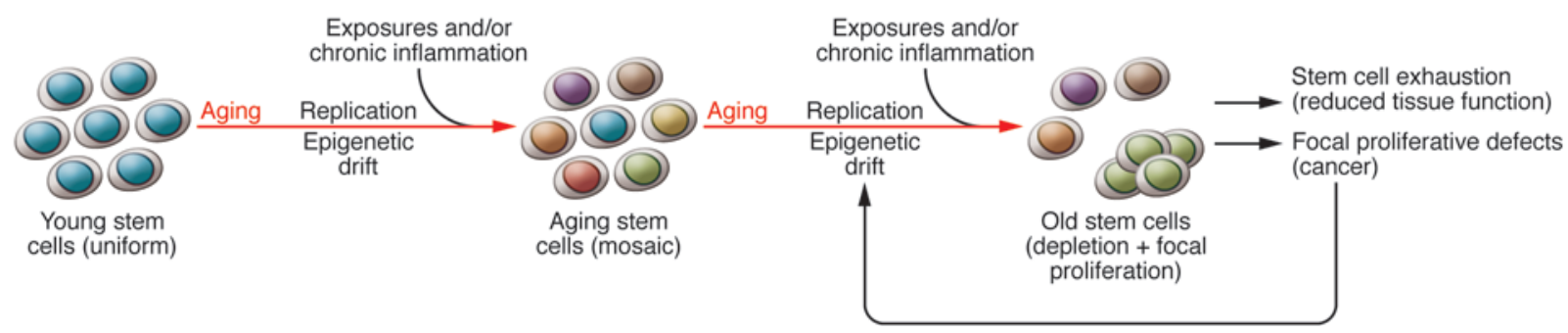

\section{Figure 2}

A model of the effects of the aging epigenome on stem cell function. Young stem cells have relatively uniform epigenomes. During aging, stem cells replicate and stochastic errors in DNA methylation maintenance introduce epigenetic mosaicism. Exposures and/or chronic inflammation accelerate this process by promoting stem cell replication (e.g., for tissue repair) or by working directly on the epigenome. Continued epigenetic mosaicism results in restricted differentiation in some stem cells, leading to stem cell exhaustion and a selective growth advantage in other stem cells, which then leads to clonal expansion and local hyperproliferation. The combination of stem cell exhaustion and clonal expansion contributes to phenotypes and diseases of aging. In turn, these proliferative and restricted differentiation phenotypes promote epigenetic drift, creating a vicious cycle that exponentially increases the rate of some diseases such as cancer.

challenge, as the easiest changes to observe are the largest shifts, which are also most likely to reflect a changing cellular milieu, differentiation, or proliferation potential. Several studies have examined the issue and found that histones, histone variants, and modified histones vary during aging in various organisms $(41,42)$. Some of the conserved changes include a global loss in histone levels, and changing these levels (and other histone modifiers) can affect life span in model organisms. Remodeling of specific marks has been described in aging cells (43-45), and it will be important to determine which of these drive the aging phenotype as opposed to reflecting secondary changes in senescent cells.

\section{Mechanisms of DNA methylation drift}

In the effort to understand mechanisms that underlie age-related DNA methylation drift, with a special focus on those changes that are clearly pathologic (e.g., drift in promoter CpG island methylation), it is important to remember the global patterns described above: drift is not directional (both hyper- and hypomethylation is seen), is not uniform across the genome, and is quite variable between individuals of the same age. These observations seem to negate a simple unifying hypothesis such as a programmed methylation change or an age-related change in writers or editors of the methylation code. On the other hand, clear patterns also emerge, such as conservation between species and a significantly higher drift in the most proliferative tissues. This raises the issue of the fidelity in replication-associated copying of DNA methylation. Using selectable markers over relatively short periods, a spontaneous error rate in methylation maintenance at promoter $\mathrm{CpG}$ islands (both gains and losses) was measured as $10^{-4}$ to $10^{-5}$ in vitro $(46,47)$. Thus, a simple explanation for methylation drift is the presence of replication-dependent errors in maintenance of epigenetic states. If one postulates that every stem cell replication event is associated with a finite chance of a DNA methylation error at a given $\mathrm{CpG}$ site, then methylation may serve as a mitotic clock (27) in the same manner as telomere shortening. This hypothesis does not account for some of the peculiarities of the process, such as genomic specificity. Studies have shown that the affected genes can be predicted to some extent using genomic features such as nearby retrotransposon density (48) (a high density is associated with fewer changes with age), occupancy by polycomb group proteins in embryonic cells (33) (with polycomb group targets showing more age-related changes), direct association with specific genomic sequences (49), and inverse association with CTCF binding sites (50). Additionally, baseline gene expression is also likely to influence this process (51). Interestingly, imprinted genes tend to show less methylation drift with age than other genomic regions (52).

To reconcile the hypothesis of a stochastic process (random errors in methylation maintenance) and the observations on genomic specificity, it is proposed that the fidelity of transmission of epigenetic patterns is variable across the genome in the same way that DNA repair efficiency varies between transcribed and nontranscribed regions (53). In this model, epigenetic errors would be repaired (or prevented) more readily in certain regions, perhaps as a result of factors such as transcription factor binding, chromatin compaction, and nucleosome density. Strong evidence for this model came from studying a length polymorphism in the RIL promoter that creates an extra SP1/ SP3 binding site (54). Individuals homozygous for the longer allele show significantly less age-related methylation at that site than those homozygous for the short allele. In transient transfection, the short allele has levels of gene expression equivalent to the long allele but is significantly more likely to show time-dependent silencing and methylation spreading. Genome-wide studies have also identified genetic variants that affect the rate of DNA methylation in cis (34), which is consistent with prior studies showing an important role for genomic polymorphisms in affecting allele-specific DNA methylation (55).

The mitotic clock hypothesis, modified to account for uneven methylation maintenance across the genome, is very consistent with both species conservation and tissue specificity of age-related methylation drift (28). It also provides a framework to study population variation. While some degree of methylation drift can be seen in all individuals past a certain age, there are genetic and acquired modifiers of the rate of methylation drift. As mentioned earlier, sequences in cis influence the degree of drift, and polymorphisms in these sequences can account for some of the population variability. Drift is also dependent on trans-acting factors such as methylases (DNMTs), demethylases (TETs), and modifiers (e.g., CTCF). It is plausible that genetic polymorphisms in the activity or targeting of these proteins contribute to population variation. The variability in methylation drift seen in inbred mice (28) indicates that there must also be genetically independent, acquired modifiers of drift. Indeed, in a study of human monozygotic twins, there was significantly greater DNA methylation discordance at 
multiple loci in older twin pairs, consistent with stochastic drift that is at least partially independent of genotype (56).

Chronic inflammation is the best-characterized acquired modifier of methylation. In the colon (57), esophagus (58), stomach (59), lung (31), and liver (60), chronic inflammation is associated with substantial methylation drift in apparently normal tissues. In a gerbil model of Helicobacter pylori infection, CpG island methylation drift required chronic inflammation (61), and while bacterial eradication reduced drift, it did not return to baseline. A high degree of drift was also seen in a mouse model of inflammatory bowel disease (62), and smoking was associated with accentuated drift in lung epithelium as well as in blood (31). The mechanism linking inflammation to drift remains unresolved. It has recently been reported that transient exposure to hypoxia (63) or reactive oxygen species (64) targets silencing complexes to DNA and could potentially promote increased DNA methylation. On the other hand, drift is bi-directional (increases at some genes, decreases at others) and cannot be accounted for solely by silencing complexes. A possible explanation for the link is indirect - it is likely that inflammation accelerates the mitotic clock by stimulating stem cell proliferation during cycles of tissue injury and repair.

Other than inflammation, acquired determinants of methylation variation remain poorly understood. There is much interest currently in a dietary epigenetic link. One carbon metabolism influences the availability of the methyl donor S-adenosyl-methionine, which could theoretically drive enzymatic DNA methylation (65). Indeed, gestational exposure to high levels of folate and vitamin $B_{12}$ can increase locus-specific DNA methylation patterns in newborn mice (66), and population variation at specific sites in newborns has been linked to month of conception in a study that shows marked seasonal variation in dietary habits (67). It is not yet known how much gestational and developmental diets influence DNA methylation drift in adults. A recent study showed that folate levels in $\mathrm{rbc}$, a measure of chronic intake, was positively correlated with methylation drift at promoter CpG islands (68) but not at repetitive sequences (21). There is also considerable interest in a potential link between environmental chemical exposures and altered epigenetics. Several naturally occurring compounds have unquestioned epigenetic effects (69), though the evidence for others is inconclusive (70). It seems likely that we are exposed to chemicals that influence epigenetic drift, but the precise nature of these chemicals and the magnitude of their effects are yet to be determined.

\section{Age-related methylation drift and physiology: altered plasticity?}

The functional consequences of age-related methylation drift remain a matter of speculation. Promoter CpG island methylation silences transcription of the gene in affected cells (4). Methylation changes elsewhere have variable, site-dependent effects on gene expression. To fully appreciate the impact of aging, one also has to consider the degree of methylation changes. Quantitative measurements reveal differences between young and old mammals on the order of 5\%-25\% at susceptible genes (28). The effects of such partial methylation depend on whether it is uniformly altered to a small degree in every cell or greatly altered in a small proportion of cells. Data suggest that, for promoter CpG islands, the latter is correct - that aged stem cells are epigenetic mosaics, with some showing no methylation at a given gene and others having a very high degree of methylation at the same gene (27). These remarkable differences would be expected to result in large differences in gene expression between different stem cells in the same tissue, assuming that the affected gene is actually active in that tissue. If one considers the thousands of genes that show age-related drift, a picture emerges in which tissues in older individuals have considerable mosaicism in gene expression profiles (summarized in Figure 2).

Epigenetic mosaicism could profoundly affect the function of aged stem cells. The loss of actual or potential gene expression limits the differentiation potential of a stem cell. As mentioned earlier, many of the genes that show age-related methylation drift are polycomb targets in embryonic stem cells and are enriched for genes functionally involved in differentiation and development $(28,62$, 71). It is likely that promoter CPG island methylation at these loci limits the plasticity of stem cells, which compromises their ability to renew healthy differentiated cells and thus contribute negatively to aged tissue function. Indeed, aged hematopoietic stem cells are an example of this plasticity defect, in that they show skewed differentiation, with more myeloid and fewer lymphoid progeny (72). There are also other ways by which epigenetic drift may compromise stem cell function. Hypermethylation may silence genes essential for renewal, leading to age-related depletion of the stem cell pool. This depletion can also result from hypomethylation and activation of genes that normally show germ cell-restricted expression (40) and are not fully recognized as self by the immune system, thus triggering autoimmune destruction of the stem cells. Overall, compromised stem cell function is a likely consequence of age-related epigenetic drift, and there is some evidence that stem cell aging is a key mechanism regulating life span (73).

A major physiologic consequence of age-related drift is the appearance of tissue-level epigenetic mosaicism. On the surface, mosaicism is not necessarily deleterious for tissue function; however, epigenetic mosaicism equates to stable gene expression variation, which in turn is a powerful engine for natural selection (74). Imagining millions of stem cells with variation affecting hundreds to thousands of genes, it is easy to see how competition would result in the extinction of some stem cells and in the expansion of others that have a selective growth advantage compared with neighboring cells (Figure 2). Evidence for this comes from the gastrointestinal tract, in which each crypt is formed by several stem cells in young individuals but only by one or two stem cells in aged mice and humans (75). Indeed, aging is accompanied by crypt expansion, and one can then observe multiple adjacent crypts all arising from the same stem cell. This is exactly what would be predicted from a model of epigenetic variation plus natural selection, and the extension of this process results in time-dependent emergence of hyperproliferative clonal lesions in affected tissues.

\section{Methylation drift and disease}

A reduced stem cell function is one of the hallmarks of aging in mammals, from loss of specific cell types to reduced capacity for tissue repair (76). It is easy to see how methylation drift can contribute to declining tissue health by constricting the plasticity of aged stem cells. Another feature of aging is the emergence of autoimmune diseases and, as discussed earlier, it is possible that age-related methylation drift contributes to this phenomenon by unmasking hidden tissue antigens or altering lymphocyte function through hypomethylation (77). It is also possible to imagine other situations in which alterations in a small number of cells could result in significant pathology, such as through induction of local inflammation or fibrosis. Thus, several age-related pathologies might have an epigenetic component. 
One of the most profound effects of epigenetic variation at the tissue level may well be aberrant focal stem cell proliferation. The two most common causes of death in humans, atherosclerotic heart disease and cancer, are both focal proliferative events. In tissues with relatively little cellular turnover and in which carcinogen exposure is relatively low and spontaneous mutations relatively few, focal proliferation itself leads to pathology, as in coronary atherosclerosis. Interestingly, aberrant methylation of genes such as $E R a$ and $E R b$ has been observed in atherosclerotic plaques $(78,79)$. In tissues regularly exposed to carcinogens, such as lung or gastrointestinal tract, the age-related clonal expansion might allow oncogenic mutations to transform cells, resulting in full-blown malignancy. Keeping in mind that most oncogenic mutations (e.g., activating KRAS mutation) lead to senescence or death of normal cells, the convergence of age-related epigenetic drift and carcinogen-induced genetic change may be the key factor in explaining the rise in incidence of cancer as humans age. Indeed, genomewide studies have now shown that cancers are characterized by coincident genetic and epigenetic damage (80).

Cancer provides a dramatic example of the interactions between aging, epigenetic drift, and disease. Cancer cells have a high degree of aberrant DNA methylation, as well as other epigenetic alterations (81). Increased promoter CPG island methylation in aging normal cells was discovered by studying normal-appearing tissues adjacent to cancer, and aging changes were suggested to be an early step in carcinogenesis (23). Approximately $70 \%-80 \%$ of the DNA methylation abnormalities in cancer can be traced to aging defects (25), and cancer itself can be seen as an accelerated aging phenomenon if one considers stem cell replication as the ultimate mark of the biologic clock. In addition to these descriptive studies, there are direct convincing data linking epigenetic defects and oncogenesis. First, some of the genes altered epigenetically in cancer are bona-fide tumor suppressor genes that cause familial cancer clusters when mutated, and there is an inverse correlation between genetic and epigenetic defects at these loci, suggesting an equivalent growth advantage regardless of the molecular defect (81). Second, writers, editors, and readers of the epigenetic code are often mutated in cancer (82), suggesting that the downstream epigenetic defects are functionally responsible for the neoplastic phenotype. Finally, in mouse models, reducing methylation in normal colon by partial deletion of $D n m t 1$ prevents the development of polyps (83), while increasing methylation by overexpression of Dnmt3b increases polyp formation (84). These data support the model that predicts that age-related methylation defects are rate limiting for the development of some preneoplastic lesions. In human carcinogenesis, cancers are often found to arise in fields of aberrant DNA methylation (85), which is also consistent with the model.

\section{Conclusions: a vicious cycle}

DNA methylation and epigenetic inheritance show a significant drift with age in mammals, most likely because of stochastic errors in epigenetic copying during stem cell replication. This drift results in gene expression mosaicism and has the potential to substantially contribute to aging phenotypes, including disease. Aging pathologies in turn accelerate methylation drift by promoting chronic inflammation and uncontrolled proliferation, thus creating a vicious cycle (Figure 2) that may explain why some diseases such as cancer increase exponentially rather than linearly with age. In addition to its potential as a physiologic marker of disease risk, it is worth considering whether the prevention of epigenetic drift might alleviate disorders and diseases associated with aging in humans.

\section{Acknowledgments}

Relevant research in the author's laboratory is supported by NIH grant CA158112 and by a grant from the Ellison Medical Foundation. The author is an American Cancer Society Clinical Research professor supported by a generous gift from the F. M. Kirby Foundation.

Address correspondence to: Jean-Pierre Issa, Fels Institute for Cancer Research and Molecular Biology, Temple University, 3307 North Broad Street, Rm. 154 - PAHB, Philadelphia, Pennsylvania 19140, USA. Phone: 215.707.4307; Fax: 215.707.1454; E-mail: jpissa@temple.edu.
1. Waddington CH. The epigenotype. Endeavour. 1942; 1:18-20.

2. Heard E, Clerc P, Avner P. X-chromosome inactivation in mammals. Annu Rev Genet. 1997;31:571-610.

3. Barlow DP. Gametic imprinting in mammals. Science. 1995;270(5242):1610-1613.

4. Bird A. DNA methylation patterns and epigenetic memory. Genes Dev. 2002;16(1):6-21.

5. Tan L, Shi YG. Tet family proteins and 5-hydroxymethylcytosine in development and disease. Development. 2012;139(11):1895-1902.

6. Razin A, Riggs AD. DNA methylation and gene function. Science. 1980;210(4470):604-610.

7. Li E, Beard C, Jaenisch R. Role for DNA methylation in genomic imprinting. Nature. 1993; 366(6453):362-365.

8. Jones PA, Taylor SM. Cellular differentiation, cytidine analogs and DNA methylation. Cell. 1980;20(1):85-93.

9. Lister R, et al. Human DNA methylomes at base resolution show widespread epigenomic differences. Nature. 2009;462(7271):315-322.

10. Jones PA. Functions of DNA methylation: islands, start sites, gene bodies and beyond. Nat Rev Genet. 2012;13(7):484-492.

11. Jenuwein T, Allis CD. Translating the histone code. Science. 2001;293(5532):1074-1080.

12. Sparmann A, van LM. Polycomb silencers control cell fate, development and cancer. Nat Rev Genet.
2006;6(11):846-856.

13. Peters $\mathrm{AH}$, et al. Partitioning and plasticity of repressive histone methylation states in mammalian chromatin. Mol Cell. 2003;12(6):1577-1589.

14. Henikoff S, Shilatifard A. Histone modification: cause or cog? Trends Genet. 2011;27(10):389-396.

15. Bird A. Perceptions of epigenetics. Nature. 2007; 447(7143):396-398.

16. Badeaux AI, Shi Y. Emerging roles for chromatin as a signal integration and storage platform. Nat Rev Mol Cell Biol. 2013;14(4):211-224.

17. Wilson VL, Jones PA. DNA methylation decreases in aging but not in immortal cells. Science. 1983; 220(4601):1055-1057.

18. Wilson VL, Smith RA, Ma S, Cutler RG. Genomic 5-methyldeoxycytidine decreases with age. J Biol Chem. 1987;262(21):9948-9951.

19. Fuke C, et al. Age related changes in 5-methylcytosine content in human peripheral leukocytes and placentas: an HPLC-based study. Ann Hum Genet. 2004;68(pt 3):196-204

20. Bollati V, et al. Decline in genomic DNA methylation through aging in a cohort of elderly subjects. Mech Ageing Dev. 2009;130(4):234-239.

21. Figueiredo JC, et al. Global DNA hypomethylation (LINE-1) in the normal colon and lifestyle characteristics and dietary and genetic factors. Cancer Epidemiol Biomarkers Prev. 2009;18(4):1041-1049.

22. Estecio MR, Issa JP. Tackling the methylome: recent methodological advances in genome-wide methylation profiling. Genome Med. 2009;1(11):106.

23. Issa JP, Ottaviano YL, Celano P, Hamilton SR, Davidson NE, Baylin SB. Methylation of the oestrogen receptor $\mathrm{CPG}$ island links ageing and neoplasia in human colon. Nat Genet. 1994;7(4):536-540.

24. Ahuja N, Li Q, Mohan AL, Baylin SB, Issa JPJ. Aging and DNA methylation in colorectal mucosa and cancer. Cancer Res. 1998;58(23):5489-5494.

25. Toyota M, Ahuja N, Ohe-Toyota M, Herman JG, Baylin SB, Issa JPJ. CpG island methylator phenotype in colorectal cancer. Proc Natl Acad Sci U S A. 1999;96(15):8681-8686

26. Waki T, Tamura G, Sato M, Motoyama T. Agerelated methylation of tumor suppressor and tumor-related genes: an analysis of autopsy samples. Oncogene. 2003;22(26):4128-4133.

27. Shibata D. Inferring human stem cell behaviour from epigenetic drift. J Pathol. 2009;217(2):199-205.

28. Maegawa S, et al. Widespread and tissue specific age-related DNA methylation changes in mice. Genome Res. 2010;20(3):332-340.

29. Beerman I, et al. Proliferation-dependent alterations of the DNA methylation landscape underlie hematopoietic stem cell aging. Cell Stem Cell. 2013;12(4):413-425.

30. Hernandez DG, et al. Distinct DNA methylation changes highly correlated with chronological age in the human brain. Hum Mol Genet. 2011; 
20(6):1164-1172

31. Christensen BC, et al. Aging and environmental exposures alter tissue-specific DNA methylation dependent upon CpG island context. PLoS Genet 2009;5(8):e1000602.

32. Gronniger E, et al. Aging and chronic sun exposure cause distinct epigenetic changes in human skin. PLoS Genet. 2010;6(5):e1000971.

33. Rakyan VK, et al. Human aging-associated DNA hypermethylation occurs preferentially at bivalent chromatin domains. Genome Res. 2010;20(4):434-439.

34. Hannum G, et al. Genome-wide methylation profiles reveal quantitative views of human aging rates. Mol Cell. 2013;49(2):359-367.

35 . Horvath S, et al. Aging effects on DNA methylation modules in human brain and blood tissue. Genome Biol. 2012;13(10):R97.

36. Lapeyre JN, Becker FF. 5-Methylcytosine content of nuclear DNA during chemical hepatocarcinogenesis and in carcinomas which result. Biochem Biophys Res Commun. 1979;87(3):698-705.

37. Kren BT, Trembley JH, Steer CJ. Alterations in mRNA stability during rat liver regeneration. Am J Physiol. 1996;270(5 pt 1):G763-G777.

38. Houseman EA, et al. DNA methylation arrays as surrogate measures of cell mixture distribution. BMC Bioinformatics. 2012;13:86.

39. Yoshihara K, et al. Inferring tumour purity and stromal and immune cell admixture from expression data. Nat Commun. 2013;4:2612.

40. Shen L, et al. Genome-wide profiling of DNA methylation reveals a class of normally methylated $\mathrm{CPG}$ island promoters. PLoS Genet. 2007;3(10):2023-2036.

41. Feser J, Tyler J. Chromatin structure as a mediator of aging. FEBS Lett. 2011;585(13):2041-2048.

42. O'Sullivan RJ, Karlseder J. The great unravelling: chromatin as a modulator of the aging process. Trends Biochem Sci. 2012;37(11):466-476.

43. Liu L, et al. Chromatin modifications as determinants of muscle stem cell quiescence and chronological aging. Cell Rep. 2013;4(1):189-204.

44. Maures TJ, Greer EL, Hauswirth AG, Brunet A. The H3K27 demethylase UTX-1 regulates C. elegans lifespan in a germline-independent, insulin-dependent manner. Aging Cell. 2011;10(6):980-990.

45. Wood JG, et al. Chromatin remodeling in the aging genome of Drosophila. Aging Cell. 2010;9(6):971-978.

46. Yates PA, Burman R, Simpson J, Ponomoreva ON, Thayer MJ, Turker MS. Silencing of mouse Aprt is a gradual process in differentiated cells. Mol Cell Biol. 2003;23(13):4461-4470.

47. Rose JA, Yates PA, Simpson J, Tischfield JA, Stambrook PJ, Turker MS. Biallelic methylation and silencing of mouse Aprt in normal kidney cells. Cancer Res. 2000;60(13):3404-3408.

48. Estecio MR, et al. Genome architecture marked by retrotransposons modulates predisposition to DNA methylation in cancer. Genome Res. 2010;20(10):1369-1382.

49. Feltus FA, Lee EK, Costello JF, Plass C, Vertino PM. Predicting aberrant $\mathrm{CPG}$ island methylation. Proc
Natl Acad Sci U S A. 2003;100(21):12253-12258.

50. Estecio MR, Gallegos J, Dekmezian M, Lu Y, Liang $\mathrm{S}$, Issa JP. SINE retrotransposons cause epigenetic reprogramming of adjacent gene promoters. Mol Cancer Res. 2012;10(10):1332-1342.

51. Takeshima H, Yamashita S, Shimazu T, Niwa T, Ushijima T. The presence of RNA polymerase II, active or stalled, predicts epigenetic fate of promoter CpG islands. Genome Res. 2009;19(11):1974-1982.

52. Gravina S, et al. High preservation of CpG Cytosine methylation patterns at imprinted gene Loci in liver and brain of aged mice. PLoS One. 2013; 8(9):e73496.

53. Hanawalt PC, Spivak G. Transcription-coupled DNA repair: two decades of progress and surprises. Nat Rev Mol Cell Biol. 2008;9(12):958-970.

54. Boumber YA, et al. An Sp1/Sp3 binding polymorphism confers methylation protection. PLoS Genet. 2008;4(8):e1000162.

55 . Kerkel K, et al. Genomic surveys by methylationsensitive SNP analysis identify sequence-dependent allele-specific DNA methylation. Nat Genet. 2008;40(7):904-908.

56. Talens RP, et al. Epigenetic variation during the adult lifespan: cross-sectional and longitudinal data on monozygotic twin pairs. Aging Cell. 2012; 11(4):694-703.

57. Issa JP, Ahuja N, Toyota M, Bronner MP, Brentnall TA. Accelerated age-related $\mathrm{CPG}$ island methylation in ulcerative colitis. Cancer Res. 2001; 61(9):3573-3577.

58. Eads CA, et al. Fields of aberrant CpG island hypermethylation in Barrett's esophagus and associated adenocarcinoma. Cancer Res. 2000;60(18):5021-5026.

59. Maekita T, et al. High levels of aberrant DNA methylation in Helicobacter pylori-infected gastric mucosae and its possible association with gastric cancer risk. Clin Cancer Res. 2006;12(3 pt 1):989-995.

60. Shen L, et al. DNA methylation and environmental exposures in human hepatocellular carcinoma. J Natl Cancer Inst. 2002;94(10):755-761.

61 . Niwa $\mathrm{T}$, et al. Inflammatory processes triggered by helicobacter pylori infection cause aberrant DNA methylation in gastric epithelial cells. Cancer Res. 2010;70(4):1430-1440.

62. Hahn MA, et al. Methylation of polycomb target genes in intestinal cancer is mediated by inflammation. Cancer Res. 2008;68(24):10280-10289.

63. Lu Y, Chu A, Turker MS, Glazer PM. Hypoxia-induced epigenetic regulation and silencing of the BRCA1 promoter. Mol Cell Biol. 2011;31(16):3339-3350.

64. O'Hagan HM, et al. Oxidative damage targets complexes containing DNA methyltransferases, SIRT1, and polycomb members to promoter $\mathrm{CPG}$ islands. Cancer Cell. 2011;20(5):606-619.

65. Mason JB. Biomarkers of nutrient exposure and status in one-carbon (methyl) metabolism. J Nutr. 2003;133(suppl 3):941S-947S

66. Waterland RA, Jirtle RL. Transposable elements: targets for early nutritional effects on epigenetic gene regulation. Mol Cell Biol. 2003;23(15):5293-5300.
67. Waterland RA, et al. Season of conception in rural gambia affects DNA methylation at putative human metastable epialleles. PLoS Genet. 2010;6(12):e1001252.

68. Wallace K, et al. Association between folate levels and CPG Island hypermethylation in normal colorectal mucosa. Cancer Prev Res (Phila). 2010; 3(12):1552-1564.

69. Spannhoff A, et al. Histone deacetylase inhibitor activity in royal jelly might facilitate caste switching in bees. EMBO Rep. 2011;12(3):238-243.

70. Chuang JC, et al. Comparison of biological effects of non-nucleoside DNA methylation inhibitors versus 5-aza-2'-deoxycytidine. Mol Cancer Ther. 2005;4(10):1515-1520.

71. Estécio MR, et al. Genome architecture marked by retrotransposons modulates predisposition to DNA methylation in cancer. Genome Res. 2010; 20(10):1369-1382.

72. Chambers SM, Shaw CA, Gatza C, Fisk CJ, Donehower LA, Goodell MA. Aging hematopoietic stem cells decline in function and exhibit epigenetic dysregulation. PLoS Biol. 2007;5(8):e201.

73. Liu L, Rando TA. Manifestations and mechanisms of stem cell aging. J Cell Biol. 2011;193(2):257-266.

74. Issa JP. Epigenetic variation and cellular Darwinism. Nat Genet. 2011;43(8):724-726.

75. Zeki SS, Graham TA, Wright NA. Stem cells and their implications for colorectal cancer. Nat Rev Gastroenterol Hepatol. 2011;8(2):90-100.

76. Signer RA, Morrison SJ. Mechanisms that regulate stem cell aging and life span. Cell Stem Cell. 2013; 12(2):152-165.

77. Richardson B. Primer: epigenetics of autoimmunity. Nat Clin Pract Rheumatol. 2007;3(9):521-527.

78. Kim J, et al. Epigenetic changes in estrogen receptor beta gene in atherosclerotic cardiovascular tissues and in-vitro vascular senescence. Biochim Biophys Acta. 2007;1772(1):72-80.

79. Post WS, et al. Methylation of the estrogen receptor gene is associated with aging and atherosclerosis in the cardiovascular system. Cardiovasc Res. 1999;43(4):985-991.

80. Cancer Genome Atlas Research Network. Genomic epigenomic landscapes of adult de novo acute myeloid leukemia. NEngl J Med. 2013;368(22):2059-2074.

81. Taby R, Issa JP. Cancer epigenetics. CA Cancer JClin. 2010;60(6):376-392.

82. Shih AH, Abdel-Wahab O, Patel JP, Levine RL. The role of mutations in epigenetic regulators in myeloid malignancies. Nat Rev Cancer. 2012; 12(9):599-612.

83. Laird PW, et al. Suppression of intestinal neoplasia by DNA hypomethylation. Cell. 1995;81(2):197-205.

84. Linhart HG, et al. Dnmt3b promotes tumorigenesis in vivo by gene-specific de novo methylation transcriptional silencing. Genes Dev. 2007; 21(23):3110-3122.

85. Shen L, et al. MGMT promoter methylation and field defect in sporadic colorectal cancer. J Natl Cancer Inst. 2005;97(18):1330-1338. 